\title{
Frequency and Distribution of Biopsied Non-Dental Plaque-Induced Gingival Diseases in a Chinese Population According to the Classification of Gingival Health and Gingival Disease/Conditions
}

\section{Xiaotian Li}

Peking University School and Hospital of Stomatology

Jianyun Zhang

Peking University School and Hospital of Stomatology

Heyu Zhang

Peking University School and Hospital of Stomatology

Tiejun Li ( $\square$ litiejun22@vip.sina.com )

Peking University School and Hospital of Stomatology

\section{Research Article}

Keywords: Non-dental plaque-induced gingival diseases, Classification of gingival health and gingival disease/conditions, epidemiology

Posted Date: March 22nd, 2021

DOI: https://doi.org/10.21203/rs.3.rs-308963/v1

License: (c) (1) This work is licensed under a Creative Commons Attribution 4.0 International License.

Read Full License 


\section{Frequency and distribution of biopsied non-dental plaque- induced gingival diseases in a Chinese population according to the classification of gingival health and gingival \\ disease/conditions}

Xiaotian Li ${ }^{1,3}$, Jianyun Zhang ${ }^{1,3}$, Heyu Zhang ${ }^{2,3^{*}}$, Tiejun $\mathrm{Li}^{1,3^{*}}$

1. Department of Oral Pathology, National Clinical Research Center for Oral Diseases, Peking University School and Hospital of Stomatology, Beijing, China

2. Central Laboratory, Peking University School and Hospital of Stomatology, Beijing, China

3. Research Unit of Precision Pathologic Diagnosis in Tumors of the Oral and Maxillofacial Regions, Chinese Academy of Medical Sciences (2019RU034), Beijing, China

*Correspondence to: Tiejun Li, litiejun22@vip.sina.com; Heyu Zhang, zhangheyu1983@sina.cn 


\begin{abstract}
Background: While inflammatory diseases such as gingivitis and periodontitis induced by dental plaque biofilms constitute the majority of gingival lesions, gingiva can also be affected by a variety of diseases that show etiologies different from bacterial biofilms. The aim of this study was to retrospectively analyze the frequency and distribution of non-dental plaque-induced gingival diseases (NDPIGDs) in a Chinese population.
\end{abstract}

Methods: A total of 6859 samples of biopsied gingival diseases during the period of 2000-2019 were obtained from the Department of Pathology, Peking University Hospital of Stomatology. Lesions were categorized by histopathological diagnosis, pathological nature and the new classification of gingival health and gingival diseases/conditions. Demographic information, such as gender, location, and age, were also analyzed.

Results: Among 6859 biopsied NDPIGD samples, the five most frequent diagnoses included oral squamous cell carcinoma (OSCC, $n=2094)$, fibrous hyperplasia $(n=2026)$, pyogenic granuloma $(n=478)$, epithelial dysplasia $(n=477)$, and epithelial hyperplasia/hyperkeratosis $(n=436)$. All types could be grouped into nine categories according to their pathological characteristics. The most frequently common biopsied NDPIGDs category was "hyperplastic lesions" ( $n=2648,38.61 \%)$, followed by “malignant neoplasms" ( $\mathrm{n}=2275,33.17 \%)$. The most common diagnosis types in each category were fibrous hyperplasia and OSCC. Of all NDPIGDs, most lesions could be 
categorized into the new classification of gingival health and gingival diseases/conditions; only $7.07 \%$ did not fit the current classification system.

Conclusions: The present study represents the first report on the frequency and distribution of biopsied NDPIGDs in a Chinese population. Unlike previous studies, the most prevalent categories were "hyperplastic lesions" and "malignant neoplasms". The proportion of "malignant neoplasms" and "oral potentially malignant disorders" was remarkably higher than in previous research. Nevertheless, the study provided epidemiological information for many NDPIGDs, which could be useful for future health policies as well as screening programs.

Key words: Non-dental plaque-induced gingival diseases, Classification of gingival health and gingival disease/conditions, epidemiology

\section{Background}

As one of the most common sites for various pathogens affecting oral health, gingiva has long been the concentration scope of many clinicians. While most lesions that occurr on oral gums are inflammatory diseases induced by dental plaque biofilm [1]. gingiva can also be affected by a variety of neoplastic or non-neoplastic diseases that show different etiologies from bacterial biofilm [2]. Accurate diagnosis is essential for better management of these lesions because of their various effects on clinical behavior and later treatment.

Non-dental plaque-induced gingival diseases (NDPIGDs) are defined as a group of lesions that are not caused by bacterial plaque and often do not disappear after dental plaque removal; however, it is emphasized that the severity of clinical manifestations 
sometimes relies on the interaction with the remaining bacterial plaque [3]. These lesions were not identified until the classification of periodontal diseases and conditions drawn up by the American Academy of Periodontology (AAP) in 1999. The system named and classified a wide range of diseases including gingival diseases induced by special bacteria, viruses and fungi, genetic gingiva lesions, gingival manifestation of system conditions, traumatic lesions, foreign body reaction, and not otherwise specified. However, as mentioned above, the gingiva can be affected by many types of nonneoplasms or neoplasms (either benign or malignant), which were not included in the 1999 classification system. A new classification scheme for periodontal and periimplant diseases and conditions was established by the European Federation of Periodontology and AAP in 2018 [4]. In this classification, some modifications were made, and neoplasms such as leukoplakia and squamous cell carcinoma were also included. From the perspective of pathologies, their etiology might be different; however, periodontists may be unaware of them due to the limited number of reported studies. Therefore, knowledge of the prevalence and distribution of these lesions could offer help in regional screening programs for oral health [5].

To date, limited studies focused on gingival lesions have been reported in different countries [6-11]. Pyogenic granuloma and fibroma constitute the majority of nonneoplastic lesions that are most likely seen in the gingiva [6-8,12]. Fibroma is the most common type among the neoplastic diseases while the proportion of oral squamous cell carcinoma (OSCC) accounts for most of all malignancies [7,12]. However, of all the research with valid references, none provide a summary concerning the epidemiology 
of NDPIGDs in a Chinese population. In addition, most of these investigations simply recorded the epidemiology of NDPIGDs despite the periodontal classification of these diseases. While one study combined the histopathological diagnosis with periodontal classification, it was mostly based on the classification of 1999 [12]. With the presence of a new classification of periodontal lesions proposed in 2018, it is essential to be aware of the epidemiology of NDPIGDs. Therefore, the aim of this study was to retrospectively analyze the frequency and distribution of NDPIGDs in a Chinese population.

\section{Methods}

This research was approved by IRB of Peking University Hospital of Stomatology, and written informed consent was obtained from all patients (Approval No. PKUSSIRB-201418116a). A retrospectively study was performed on gingival biopsies obtained from the Department of Pathology, Peking University Hospital of Stomatology, and was in accordance with the ethical standards laid down in the 2008 Declaration of Helsinki and its later amendments. Only records with adequate case histories were considered for the investigation purposes. In total, 6859 biopsies during the period of 2000-2019 were collected for this study. Data regarding age, sex, type, location, and histopathological diagnoses of the lesions were collected from each subject. The cases were included in the project if they affected the gingiva with adequate and definite pathological information. The ambiguous sections were reviewed and confirmed by an experienced oral pathologist (TL). Cases were excluded if they had salivary gland diseases and intraosseous lesions that extended to the gingiva. 
Nonspecific inflammatory diseases were also excluded if they could not be distinguished from gingivitis or periodontitis. Data entry was performed by using Microsoft Excel software.

\section{Results}

The number and frequency of the biopsied samples are summarized in Table 1. Among a total of 6859 biopsied NDPIGD samples, the five most frequent types of all cases included OSCC $(n=2094,30.53 \%)$, fibrous hyperplasia $(n=2026,29.54 \%)$, pyogenic granuloma $(n=478,6.97 \%)$, epithelial dysplasia $(n=477,6.95 \%)$ and epithelial hyperplasia/hyperkeratosis ( $n=436,6.36 \%)$. Specifically, OSCC ranked at the top of all NDPIGDs, indicating that OSCC was the most unpleasant lesion encountered in the gingiva. The sixth most frequent diagnosis as well as the most common type of autoimmune disorder was pemphigoid $(n=298)$, which accounted for $4.34 \%$ of all lesions. Lesions with more than 50 cases also included lichen planus ( $n=290,4.23 \%$ ), pemphigus $(n=86,1.25 \%)$, malignant melanoma $(n=86,1.25 \%)$, verruciform xanthoma $(\mathrm{n}=84,1.22 \%)$, papilloma $(\mathrm{n}=79,1.15 \%)$ and melanotic macules $(\mathrm{n}=51,0.74 \%)$.

In order to classify these different types of NDPIGDs into more specific categories, we divided the cases types into several groups according to their pathological nature. As shown in Table 2, the most frequently observed biopsied lesions were "hyperplastic lesions" (n=2648), reaching up to $38.61 \%$ of all NDPIGDs. In "hyperplastic lesions", the largest proportion was fibrous hyperplasia, followed by pyogenic granuloma. Due to the large number of OSCC cases, "malignant neoplasms" ranged from the top two of all biopsied samples, with a percentage of $33.17 \%$. The third and fourth most 
common categories were "oral potentially malignant disorders" (OPMD, n=1214, $17.7 \%)$ and "autoimmune disorders" $(\mathrm{n}=384,5.6 \%)$. Among the OPMDs, those characterized by epithelial dysplasia and hyperplasia/hyperkeratosis were the most commonly encountered; of these "autoimmune disorders", the largest number of biopsied samples was performed on pemphigoid. "Benign tumors" were also usually found on the oral gum, and papilloma seemed to be the most common. When it comes to "genetic lesions", it included only one diagnosed type named hereditary gingival fibromatosis, with an occurrence of $0.58 \%$. "Genetic lesions" was followed by "allergic reaction diseases" and "infectious diseases", which included only four cases in total. There were still some diagnosed types that could not be allocated into the above eight categories, including melanotic macules, exogenous pigmentation, vascular malformations, hemangioma and granuloma.

Next, we summarized the demographic information including gender, location, and age of all the categories based on the pathological nature. As shown in Figure 1A, females showed a higher prevalence (59.64\%) than males (40.36\%). All categories were more common in females, except benign neoplasms, which were more frequently observed in males. "Hyperplasia lesions" were the most common category for females and the second most frequent category for males. "Malignant neoplasms" followed and were found to have a high prevalence in females. "OPMDs" and "autoimmune disorders" both exhibited significant differences between genders, with a higher number of samples in females than in males. "Benign neoplasms", in contrast, were more commonly observed on males. Of the 40 biopsied genetic lesion samples, 18 cases 
were male and 22 were females. Due to the limited number of "allergic reaction diseases" category and "infectious diseases" category, gender differences were not statistically analyzed.
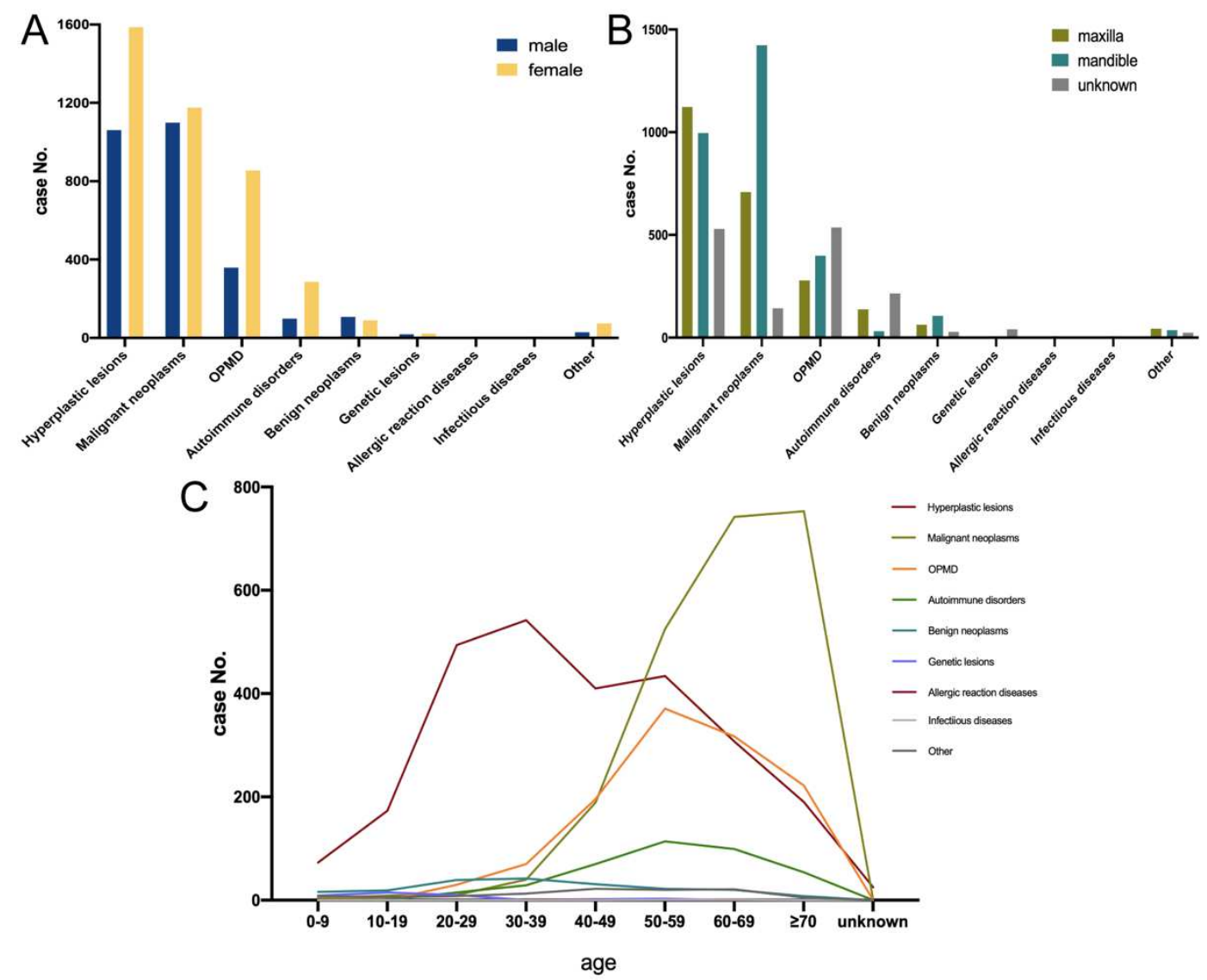

Figure 1. Demographic information of all NDPIGDs based on pathological characteristics. (A) Bar chart showing gender distribution of biopsied NDPIGDs. (B) Bar chart showing location distribution of biopsied NDPIGDs. (C) Point-fold line chart illustrating the age distribution of biopsied NDPIGDs.

The distribution of location is also summarized. As shown in Figure 1B, different categories of NDPIGDs showed an absolute location preference. "Hyperplasic lesions" had a slightly higher rate in the maxilla $(n=1123)$ than in the mandible $(n=996)$. While "malignant neoplasms" cases were more likely to occur in mandible $(n=709)$, with 
almost twice as many as than in the maxilla $(\mathrm{n}=1423)$. Apart from a number of OPMD cases that had no location information reported, the remaining cases were more likely to be obtained from the mandibular gingiva $(\mathrm{n}=537)$, in comparison with the maxillary gingiva ( $\mathrm{n}=399)$. "Autoimmune disorders" were more frequent in the maxilla $(\mathrm{n}=138)$, while "benign neoplasms" were located slightly more in the mandible $(n=106)$ compared with the maxillary gums $(n=63)$. Specifically, hereditary gingival fibromatosis affected the entire gingiva despite location prevalence. Therefore, we did not divide the biopsied samples into specific gingiva locations. "Allergic lesions and "infectious diseases" were also not analyzed due to the small number of samples.

As shown in Figure 1C, biopsied NDPIGDs were also diagnosed within a wide age range, from 0 to 86 years old. "Hyperplasia lesions" were observed in all age groups, with the highest prevalence in the age range of 20-59 years. "Malignant neoplasms" showed a prevailing concentration in elderly patients of 50-69 years old. A similar conclusion could also be drawn in the "OPMD" category, showing that the majority of these cases were diagnosed between 30-69 years old and exhibited a peak incidence among the patients in the age group of 50-59 years old. "Autoimmune disorders" were not frequently diagnosed in patients under the age of 40 , and subjects in the age group of 40-70 years were the most prevalent. "Benign neoplasms" were not specifically diagnosed in a certain age of the eight age groups. In contrast to the categories mentioned above, "genetic lesions" were mostly diagnosed under the age of 29 years. "Allergic reaction diseases", "infectious diseases" and other non-divided samples were scattered in all age groups. 
We finally classified all NDPIGDs into groups according to the new classification of gingival health and gingival diseases/conditions approved in 2018. As shown in Table 3, hereditary gingival fibromatosis was classified into the "genetic/developmental disorders" category; tuberculosis was considered a "specific infections"; pemphigoid, lichen planus, pemphigus, plasma cell gingivitis and granuloma were categorized into "inflammatory and immune conditions"; the "reactive processes" category included fibrous hyperplasia, pyogenic granuloma and peripheral giant cell granuloma; the majority of this classification category was "neoplasms", which included OSCC, epithelial dysplasia, epithelia hyperplasia/hyperkeratosis and malignant lymphoma; "gingival pigmentation" included melanotic macules and exogenous pigmentation. However, there were numerous types that could not be classified according to the new system and were grouped with "not otherwise specified". This category included malignant melanoma, verruciform xanthoma, papilloma, peripheral ameloblastoma, and another 23 types of diagnosis, most of which were malignant/benign neoplasms with a frequency of $7.07 \%$.

\section{Discussion}

Even though the majority of gingival diseases are caused by bacterial biofilms, it should also be borne in mind that numerous types of lesions with different etiological factors could also affect the gingival health, or even become life-threatening [12]. These lesions were not initially induced by dental pathogens; however, the outcomes of these lesions are also largely influenced by bacterial plaque. In this study, we carried out a retrospective study to analyze the frequency and distribution of NDPIGDs over the past 
20 years in a Chinese population. We adjusted and compared the current results to the new classification of gingival health and gingival diseases/conditions established in 2018.

The biopsied NDPIGDs in this study consisted of 43 types according to their pathological diagnosis. We then categorized them into nine groups based on their pathological characteristics. From the most common lesions to the least common were hyperplastic lesion, malignant lesions, OPMDs, autoimmune disorders, benign neoplasms, genetic lesions, allergic reaction diseases, infectious diseases, and other diseases that cannot be categorized into the former groups.

Previous studies regarding the classification of biopsied gingival lesions were mainly divided into three categories: non-neoplastic lesions, benign lesions, and malignant lesions $[2,6,7]$, and the majority of biopsied samples showed non-neoplastic lesions. Consistent with their reports, our statistical results also showed that the largest number of NDPIGDs were hyperplastic lesions, with $38.61 \%$ of all cases. Among them, the most common diagnosis type was fibrous hyperplasia, which accounted for nearly $30 \%$ of all cases. As important parts of the epulis, pyogenic granuloma and peripheral giant cell granuloma also showed relatively high frequencies. In particular, females showed a higher prevalence of hyperplastic lesions compared with males, which less frequently occurred in the mandible than in the maxilla. In our results, hyperplastic lesions widely ranged between and 20-59 years of age, with an incidence peak between and 30-39 years; however, this is not in complete agreement with previous studies. In a similar study, the highest frequency appeared in patients between and 60-69 years [2], while 
another two studies pointed out that the majority of biopsied samples were between 3039 years and 20-29 years [5,13]. We assumed that the inconsistency was possibly caused by different criteria as well as pathological diagnosis habits. Furthermore, one of these studies merged reactive hyperplastic lesions and other inflammatory diseases into one group.

The largest inconsistency of the biopsied NDPIGDs were "malignant neoplasms" category. In past research, the proportion of malignancies on the gingiva was reported to be $1-8 \%[2,5,11]$. However, malignant neoplastic lesions represented up to $33.14 \%$ of all gingival lesions in our study. This high incidence was significantly different from that in early reports. Among them, Layfield et al represented the most comprehensive biopsied gingival lesions research that collected a total of 30,056 samples over a period of 24 years. In their study, the frequency of malignant neoplasms was as low as $1.4 \%$. Such a huge difference demonstrated the difference in prevalence between Easterners and Westerners. Notably, when considering the distribution of the malignancies, the most prevalent neoplasm reported in our research and previous studies was the same, OSCC $[14,15]$, which accounted for $30.53 \%$ of all samples.

The former category was then followed by "OPMDs", "autoimmune disorders", and "benign neoplasms". The category "OPMD" was defined as a significant group of mucosal disorders that may precede the diagnosis of OSCC [16], which included oral leukoplakia, oral erythroplakia, oral lichen, and newly added oral lichenoid lesions. Depending on the presence or absence of epithelial dysplasia, oral leukoplakia was divided into epithelial hyperplasia/hyperkeratosis and epithelial dysplasia, with 
frequency of $6.36 \%$ and $6.95 \%$, respectively. In this study, lichen planus and oral lichenoid lesions were placed into the OPMD category. In total, the total number of OPMDs reached 1214 , accounting for $17.7 \%$ of all NDPIGD cases. This ratio was remarkably higher than that previously reported $[11,17]$.

The "autoimmune disorders" category was firstly put forward in our work and pemphigoid and pemphigus were categorized into the group. In former studies, pemphigoid and pemphigus were not specifically mentioned, either due to the low frequency or they were classified into "mucocutaneous disorders" [11]. However, the two lesions in our work account for $5.6 \%$ of all samples, and we believed "mucocutaneous disorders" was ambiguous for describing the type, as many other diseases like oral leukoplakia might be confused. Benign neoplasms, on the other hand, are not commonly seen in oral gums. Of all 6859 biopsied samples, only 197 cases of different diagnosis types were observed. While malignant neoplasms reached a peak incidence in those aged over 60 years, benign tumors were distributed separately.

Hereditary gingival fibromatosis is a frequently common genetic disease seen in the periodontal clinic. Because the present research is mainly concentrated on biopsied cases, the frequency $(0.58 \%)$ might be largely underestimated compared to the actual incidence in clinical procedures. Patricia's team [11] classified papilloma into "infectious lesions" while we placed papilloma into benign neoplasms and tuberculosis was the only diagnosed type in the "infectious lesions" group. Plasma cell gingivitis cases were also found on two female patients. 
Etiologies of gingival diseases were first introduced into the classification of periodontal diseases and conditions in 1999 [3]. This classification added gingival disease for the first time and divided them into two subcategories based on the initial factor, that is, dental plaque-induced gingival diseases and non-plaque-induced gingival diseases. Patricia et al distributed the biopsied non-plaque-induced gingival lesions based on the 1999 classification of periodontal diseases and conditions. However, as they presented, over $90 \%$ of biopsied gingival lesions could not be categorized according to this classification, including the most prevalent groups (hyperplastic lesions and malignant neoplasms) [11]. To a large extent, these results reflect the shortcomings of this classification system.

To the best of our knowledge, the present study represents the first report on the frequency and distribution of biopsied NDPIGDs within a Chinese population. This is also the first study to compare the pathological nature classification and the latest 2018 classification of gingival diseases. The epidemiology of the pathological diagnosis of NDPIGDs shared numerous similarities as well as certain differences with previous reports. Specifically, when categorizing pathologically diagnosed biopsied lesions with the pathological nature classification and the new classification of gingival diseases, there were some discrepancies. Lichen planus and lichenoid lesions were grouped into the "OPMD" category, however, lichen planus was grouped into "inflammatory and immune conditions" according to the new classification of gingival diseases, and lichenoid lesions were not specifically categorized. These results demonstrated the inconsistencies between the two classification systems, which may confuse clinicians' 
judgments. It should be noted that the classification system must be helpful for clinicians in early diagnosis and subsequent treatments. A classification system with more specific categories that based on prevalence, specificity and mortality is highly recommended.

However, there are still some limitations that require further investigation. First, our samples were all from the same hospital and the study is a single-center retrospective research. Second, we lack complete clinical and demographic information for many cases. In addition, our study only investigated the biopsied samples with definite pathological diagnosis; those gingival lesions that can be recognized through clinical performance without biopsy were not included into the research. Overall, the present research represented the first report of the distribution and frequency of biopsied NDPIGDs in a Chinese population as well as the first report to compare the prevalence with 2018 gingival diseases.

\section{Conclusions}

In conclusion, our retrospective study demonstrated that hyperplastic lesions and malignant neoplasms were the most common biopsied NDPIGDs in a Chinese population. Certain differences did exist among different classification systems, and future classification schemes could be improved by taking more comprehensive information (i.e., prevalence, mortality, and specificity) into consideration.

\section{Declaration}

\section{Ethics approval and consent to participate}


This research was approved by IRB of Peking University Hospital of Stomatology, and written informed consent was obtained from all patients (Approval No. PKUSSIRB201418116a).

\section{Consent for publication}

No applicable.

\section{Availability of data and materials}

The datasets used and/or analyzed during the current study are not publicly available due to the absence of a public storage online platform in our hospital, but are available from the corresponding author on reasonable request.

\section{Competing interests}

The authors declare that they have no competing interests.

\section{Funding}

This research was supported by research grants from the National Natural Science Foundation of China (81671006, 81300894) and CAMS Innovation Fund for Medical Sciences (2019-I2M-5-038).

\section{Authors' contributions}

TL and HZ designed the experiments. XL and JZ executed the experiments. TL and JZ reviewed and confirmed the sections. XL analyzed the data and wrote the manuscript. 
TL and HZ made critical revision. All authors gave final approval and are accountable for the work.

\section{Acknowledgements}

No applicable.

\section{Reference}

1. Scannapieco FA, Gershovich E. The prevention of periodontal disease-An overview. Periodontol 2000. 2020; 84: 9-13.

2. Carbone M, Broccoletti R, Gambino A, Carrozzo M, Tanteri C, Calogiuri PL, et al. Clinical and histological features of gingival lesions: A 17-year retrospective analysis in a northern Italian population. Med Oral Patol Oral Cir Bucal. 2012;1: e555-61.

3. Holmstrup P. Non-plaque-induced gingival lesions. Ann Periodontol. 1999; 4: 2031.

4. Chapple ILC, Mealey BL, Van Dyke TE, Bartold PM, Dommisch H, Eickholz P, et al. Periodontal health and gingival diseases and conditions on an intact and a reduced periodontium: Consensus report of workgroup 1 of the 2017 World Workshop on the Classification of Periodontal and Peri-Implant Diseases and Conditions. J Periodontol. 2018;89 Suppl 1: S74-S84.

5. Layfield LL, Shopper TP, Weir JC. A diagnostic survey of biopsied gingival lesions. J Dent Hyg. 1995; 69: 175-9. 
6.Bhari Sharanesha Manjunatha, Rakesh Sutariya, V Nagamahita, Bhavik Dholia, Vandana Shah. Analysis of gingival biopsies in the Gujarati population: a retrospective study. Cancer Res Ther. 2014; 10:1088-92.

7. Kavitha P Kamath, M Vidya, Pradeep S Anand. Biopsied lesions of the gingiva in a southern Indian population - a retrospective study. Oral Health Prev Dent. 2013; 11: $71-9$

8. Kansky AA, Didanovic V, Dovsak T, Brzak BL, Pelivan I, Terlevic D. Epidemiology of oral mucosal lesions in Slovenia. Radiol Oncol. 2018; 52:263-266.

9. Gambino A, Carbone M, Broccoletti R, Carcieri P, Conrotto D, Carrozzo M, et al. A report on the clinical-pathological correlations of 788 gingival lesion. Med Oral Patol Oral Cir Bucal. 2017; 22: e686-e693.

10. Carbone M, Broccoletti R, Gambino A, Carrozzo M, Tanteri C, Calogiuri PL, et al. Clinical and histological features of gingival lesions: A 17-year retrospective analysis in a northern Italian population. Med Oral Patol Oral Cir Bucal. 2012; 17: e555-61.

11. Hernández-Ríos P, Espinoza I, Salinas M, Rodríguez-Castro F, Baeza M, Hernández M. Distribution of biopsied non plaque-induced gingival lesions in a Chilean population according to the classification of periodontal diseases. BMC Oral Health. 2018; 18:112.

12. Armitage GC. Development of a classification system for periodontal diseases and conditions. Ann Periodontol. 1999; 4:1-6.

13. Ababneh KT. Biopsied gingival lesions in northern Jordanians: A retrospective analysis over 10 years. Int J Periodontotics Restorative Dent. 2006; 26: 387-93. 
14. Shamim T, Varghese VI, Shameena PM, Sudha S. A retrospective analysis of gingival biopsied lesions in South Indian population: 2001-2006. Med Oral Patol Oral Cir Bucal. 2008;13: E414-8.

15. Dhanuthai K, Rojanawatsirivej S, Subarnbhesaj A, Thosaporn W, Kintarak S. A multicenter study of oral malignant tumors from Thailand. J Oral Maxillofac Pathol. 2016; 20: 462-6.

16. Warnakulasuriya, S., Johnson, N. W., \& van der Waal, I. Nomenclature and classification of potentially malignant disorders of the oral mucosa. Journal of Oral Pathology and Medicine. 2007; 36: 575-580.

17. Carvalho Mde V, Iglesias DP, do Nascimento GJ, Sobral AP. Epidemiological study of 534 biopsies of oral mucosal lesions in elderly Brazilian patients. Gerodontology. 2011; 28:111-5. 
Table 1 The number and frequency of different biopsied NDPIGDs according to diagnosis.

\begin{tabular}{|c|c|}
\hline Type & Total no. $(\%)$ \\
\hline Oral squamous cell carcinoma & $2094(30.53)$ \\
\hline Fibrous hyperplasia & $2026(29.54)$ \\
\hline Pyogenic granuloma & $478(6.97)$ \\
\hline Epithelial dysplasia & $477(6.95)$ \\
\hline Epithelia hyperplasia/Hyperkeratosis & $436(6.36)$ \\
\hline Pemphigoid & $298(4.34)$ \\
\hline Lichen planus & $290(4.23)$ \\
\hline Pemphigus & $86(1.25)$ \\
\hline Malignant melanoma & $86(1.25)$ \\
\hline Verruciform xanthoma & $84(1.22)$ \\
\hline Papilloma & $79(1.15)$ \\
\hline Melanotic macules & $51(0.74)$ \\
\hline Hereditary gingival fibromatosis & $40(0.58)$ \\
\hline Peripheral ameloblastoma & $40(0.58)$ \\
\hline Malignant lymphoma & $33(0.48)$ \\
\hline Peripheral giant cell granuloma & $32(0.47)$ \\
\hline Drug-induced gingival enlargements & $28(0.41)$ \\
\hline Peripheral odontogenic fibroma & $28(0.41)$ \\
\hline Exogenous pigmentation & $28(0.41)$ \\
\hline
\end{tabular}


Soft tissue sarcoma

Spindle cell sarcoma

Nevus

Vascular malformations

Lichenoid lesion

Metastatic carcinoma

Myofibroma

Hemangioma

Malignant peripheral neurolemmoma

Neurofibromatosis

Neurolemmoma

Inflammatory myofibroblastic tumor

Leukemia

Myoepithelial carcinoma

Fibrous histiocytoma

Plasma cell gingivitis

Tuberculosis

Lipoma

Solitary fibrous tumor

Congenital epulis of the newborn

Leiomyoma

Granuloma
$24(0.35)$

$22(0.32)$

$21(0.31)$

$18(0.26)$

$11(0.16)$

$6(0.09)$

$5(0.07)$

$5(0.07)$

$4(0.06)$

$4(0.06)$

$3(0.04)$

$3(0.04)$

$2(0.03)$

$2(0.03)$

$2(0.03)$

$2(0.03)$

$2(0.03)$

$2(0.03)$

$2(0.03)$

$1(0.01)$

$1(0.01)$

$1(0.01)$ 
Neurofibrosarcoma

$1(0.01)$

Low-grade malignant myofibroblastoma $1(0.01)$

Total

$6859(100)$ 
Table 2 The number and frequency of different biopsied NDPIGDs according to pathological nature.

\begin{tabular}{|c|c|c|}
\hline Pathological nature & Histopathological diagnosis & Total n $(\%)$ \\
\hline \multirow[t]{4}{*}{ Hyperplasic lesions } & Fibrous hyperplasia, Pyogenic granuloma, & $2648(38.61)$ \\
\hline & Verruciform xanthoma, Peripheral giant cell & \\
\hline & Drug-induced & \\
\hline & enlargements & \\
\hline \multirow[t]{7}{*}{ Malignant neoplasms } & Oral squamous cell carcinoma, Malignant & $2275(33.17)$ \\
\hline & melanoma, Malignant lymphoma, Soft tissue & \\
\hline & sarcoma, Spindle cell sarcoma, Metastatic & \\
\hline & carcinoma, & \\
\hline & neurolemmoma, Leukemia, Myoepithelial & \\
\hline & carcinoma, $\quad$ Low-grade & \\
\hline & myofibroblastoma, Neurofibrosarcoma & \\
\hline Oral potentially & Epithelial dysplasia, Epithelia hyperplasia/ & $1214(17.7)$ \\
\hline malignant disorders & Hyperkeratosis, Lichen planus, Lichenoid lesion & \\
\hline Autoimmune & Pemphigoid, Pemphigus & $384(5.6)$ \\
\hline \multicolumn{3}{|l|}{ disorders } \\
\hline \multirow[t]{3}{*}{ Benign neoplasms } & Papilloma, Peripheral ameloblastoma, Peripheral & $191(2.78)$ \\
\hline & odontogenic fibroma, Nevus, Myofibroma, & \\
\hline & Neurofibromatosis, & \\
\hline
\end{tabular}


histiocytoma, Lipoma, Solitary fibrous tumor,

Congenital epulis of the newborn, Leiomyoma

Genetic lesions $\quad$ Hereditary gingival fibromatosis $40(0.58)$

$\begin{array}{llr}\text { Allergic reaction } & \text { Plasma cell gingivitis } & 2(0.03) \\ \text { diseases } & & \\ \text { Infectious diseases } & \text { Tuberculosis } & \\ \text { other } & \text { Melanotic macules, Exogenous pigmentation, } 103(1.50) \\ & \text { Vascular malformations, Hemangioma, } \\ & & \end{array}$

Total

$6859(100)$ 
Table 3 The number and frequency of different biopsied NDPIGDs according to new classification scheme for periodontal and peri-implant diseases and conditions.

New classification scheme Histopathological diagnosis Total n (\%)
for periodontal and peri-
implant diseases and
conditions

Genetic/ developmental Hereditary gingival fibromatosis $\quad 40(0.58)$

disorders

$\begin{array}{ll}\text { Specific infection } \quad \text { Tuberculosis } & 2(0.03)\end{array}$

Inflammatory and immune Pemphigoid, Lichen planus, Pemphigus, 677 (9.87)

conditions $\quad$ Plasma cell gingivitis, Granuloma

Reactive processes $\quad$ Fibrous hyperplasia, Pyogenic $2536(36.94)$

granuloma, Peripheral giant cell

granuloma

Neoplasms $\quad$ Oral squamous cell carcinoma, Epithelial 3040 (44.32)

dysplasia, Epithelia hyperplasia/

Hyperkeratosis, Malignant lymphoma

Gingival pigmentation Melanotic macules, Exogenous $79(1.15)$

pigmentation

Not otherwise specified Malignant melanoma, Verruciform 485 (7.07)

xanthoma, Papilloma, Peripheral

ameloblastoma, Drug-induced gingival 
enlargements, Peripheral odontogenic

fibroma, Soft tissue sarcoma, Spindle cell

sarcoma, Nevus, Vascular

malformations, Lichenoid lesion,

Metastatic carcinoma, Myofibroma,

Hemangioma, Malignant peripheral

neurolemmoma, Neurofibromatosis,

Neurolemmoma, Inflammatory

myofibroblastic tumor, Leukemia,

Myoepithelial carcinoma, Fibrous

histiocytoma, Lipoma, Solitary fibrous

tumor, Congenital epulis of the newborn,

Leiomyoma, Neurofibrosarcoma, Low-

grade malignant myofibroblastoma 
Figures

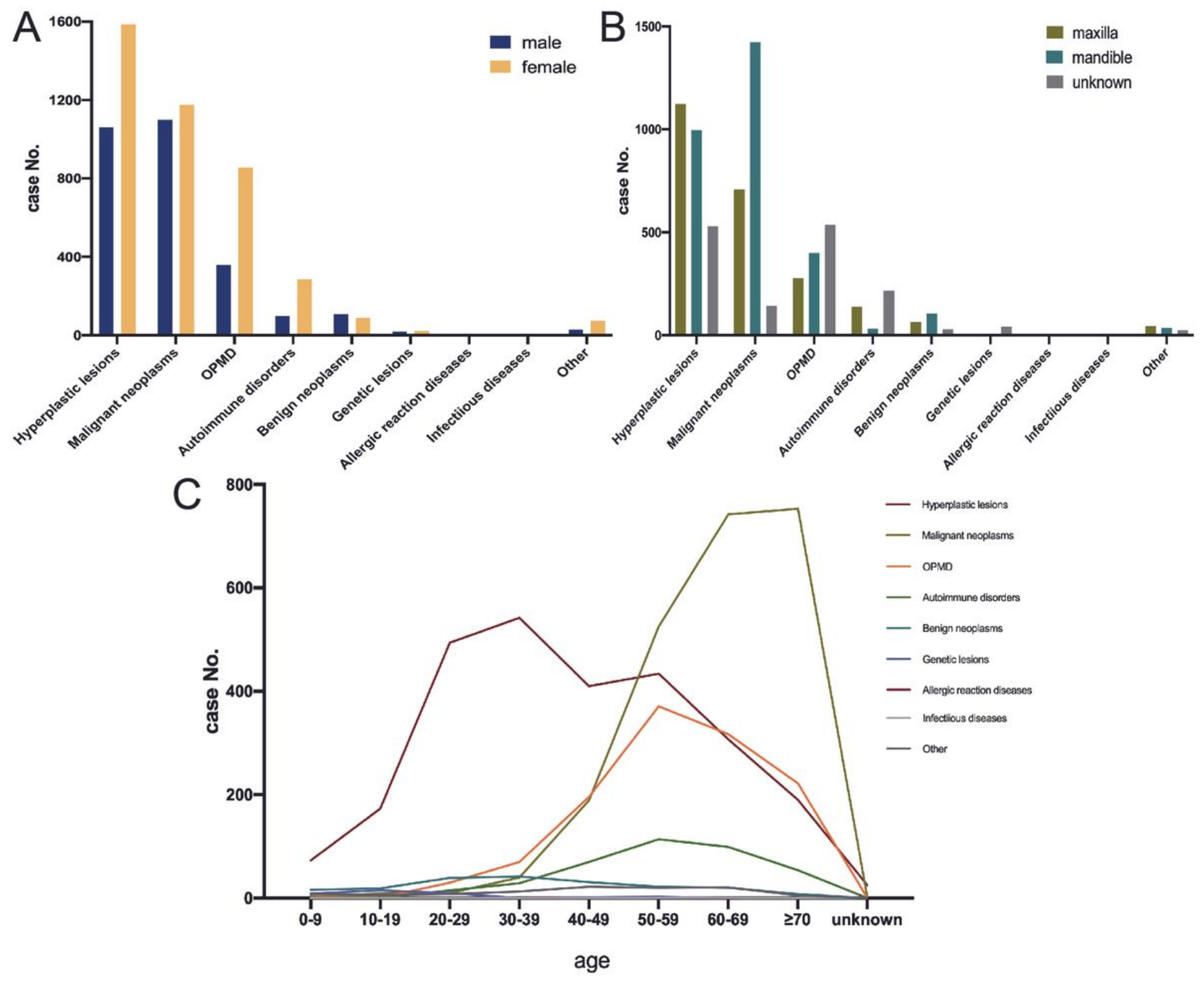

Figure 1

Demographic information of all NDPIGDs based on pathological characteristics. (A) Bar chart showing gender distribution of biopsied NDPIGDs. (B) Bar chart showing location distribution of biopsied NDPIGDs. (C) Point-fold line chart illustrating the age distribution of biopsied NDPIGDs. 